Sharif University of Technology
Scientia Iranica
Transactions E: Industrial Engineering
wCIENTIA

\title{
A multi-objective multi-state degraded system to optimize maintenance/repair costs and system availability
}

\author{
A. Salmasnia ${ }^{a, *}$, E. Ameri ${ }^{\mathrm{b}}$, A. Ghorbanian ${ }^{\mathrm{b}, 1}$ and H. Mokhtari ${ }^{\mathrm{c}}$ \\ a. Department of Industrial Engineering, Faculty of Engineering and Technology, University of Qom, Qom, Iran. \\ b. Department of Industrial Engineering, Faculty of Engineering, Tarbiat Modares University, Tehran, Iran. \\ c. Department of Industrial Engineering, Faculty of Engineering, University of Kashan, Kashan, Iran.
}

Received 17 May 2015; received in revised form 12 December 2015; accepted 9 April 2016

\author{
KEYWORDS \\ Multi-state system; \\ Markov process; \\ Multi-objective \\ optimization; \\ Preventive \\ maintenance; \\ Minimal repair; \\ Desirability function.
}

\begin{abstract}
In this study, a multi-state degraded system is studied, where status of system is continuously degrading over time. As time progresses, system may either deteriorate gradually and go to lower performance state, or it may fail suddenly. If the system fails, some repairs are carried out to restore the system to the previous state. When the inspections reveal that the system has reached its last acceptable state, a PM is carried out to restore the system to the higher performance states. The goal is to find the optimal PM level, so that the mean availability of the system is maximized and the total cost of the system is minimized. In this regard, Markov process is employed to represent different states of system. An integrated optimization approach is also suggested based on the desirability function of statistical approach. The suggested aggregation method is robust to the potential dependency between the total cost and the mean availability. It also ensures that both objective functions fall in decision-maker's acceptable region. In order to show the efficiency of the proposed approach, a numerical example is presented and analyzed.

(C) 2017 Sharif University of Technology. All rights reserved.
\end{abstract}

\section{Introduction}

A degraded system is an operating one whose status is degrading over time, and this degradation may affect its performance $[1,2]$. This phenomenon occurs in many real-world applications like transmission line networks, chemical processes, manufacturing industries, etc. In recent years, system availability has received extensive interest in many real-world systems such as computer [3] and communication systems [4], transporta-

1. Present address: Department of Industrial Engineering, Esfarayen University of Technology, North Khorasan, Iran.

*. Corresponding author. Tel.: 02532103585;

E-mail addresses: a.salmasnia@qom.ac.ir (A. Salmasnia); ameriehsan@yahoo.com (E.Ameri);

ali.ghorbanian83@gmail.com (A. Ghorbanian);

mokhtari_ie@kashanu.ac.ir (H. Mokhtari) tion systems [5], oil/gas production systems [6], etc. Most of studies considered two states for each system, so that the system was either working, or it completely failed. This is called a binary-state system [7]. However, in many real-world cases, this binary-state assumption may not be sufficient [8]. System may have more than two levels of performance varying from perfect functioning to complete failure. In other words, system may perform at various intermediate states between working perfectly and total failure $[9,10]$. The presence of degradation is a common situation in which a system should be considered to be a Multi-State System (MSS). The gradual deterioration of system may be due to several reasons including the operating hours, the environmental conditions of the equipment, the failures of non-essential components, and the number of random shocks on the system. Basic concepts of multi- 
stage systems have been introduced in [11-14]. Also, two pieces in literature review on multi-state systems can be found in $[15,16]$. A variety of approaches used in multi-state systems follow different perspectives which include:

1. The structure function approach in which Boolean models have been used for the multi-valued cases (e.g., in [12-14]);

2. The Monte Carlo simulation technique (e.g., in [17]);

3. The Markov process approach (e.g., in $[18,19]$ );

4. The method of Universal Moment Generating Function (UMGF) (e.g., see [20,21]).

One of the possible ways to improve a multi-state degraded system and to increase its availability is the use of Preventive Maintenance (PM). This will dramatically reduce the costs of stopping the system [9]. Perfect PM aims to make the system as good as before starting to work, while imperfect PM may bring the system back to an intermediate state between the current state and the perfect functioning state [22]. In some papers like [23,24], a multi-state system with the state-dependent cost was investigated. In [25], a deteriorating repairable multi-state system with an imperfect policy was offered based on the number of system failures. In [26], a monotone process maintenance model for a multi-state system was developed. An analytical approach based on the failure number of the system was used to determine the optimal replacement policy. Soro et al. [27] suggested that if the system reaches the last acceptable degraded state, it is brought back to one of the states with higher efficiency employing preventive maintenance. Nourelfath et al. [28] formulated a joint redundancy and imperfect preventive maintenance planning optimization model for series-parallel multi-state degraded systems.

The majority of solution approaches used in the literature are single-objective approaches. In other words, most of the existing approaches concentrated only on maximizing system availability, and assumed that there is no cost limitation [29-37], or considered the cost limitation only as a constraint [38-40]. However, it is usually impossible for a single-objective approach to represent a practical problem truly. Moreover, we can find several reliability/availability approaches to address different problems [41-46].

In this paper, a multi-state degraded system is considered. Even if the system deteriorates continuously, it is assumed that this process is done in a finite number of discrete states, and Markov chain is used to show the different states. It is also assumed that preventive maintenance can be performed in several different levels, varying from minor maintenance to major maintenance. A minor PM restores the system to the previous degraded state, while a major PM restores it to the "as good as new" state. The goal is to find the optimal PM level, so that the mean availability of the system is maximized, and the total cost of the system is minimized during the useful lifetime of the system. The suggested integration approach includes the following features:

1. It is robust to the potential dependences between the total cost and the mean availability;

2. It aims to identify the settings of the decision variables to maximize the degree of overall satisfaction with respect to total cost and mean availability.

The remainder of the paper is organized as follows. In Section 2, the assumptions are presented and the multistate degraded system under study is described. In Section 3, the availability and cost functions are represented and a "minimum" operator for aggregating them is employed. In Section 4, an illustrative example is presented. Conclusions and some remarks are provided in Section 5.

\section{Model description}

Initially, there is a system which is in its perfect functioning state (state 1). Over time as shown in Figure 1, two things may happen: the system may

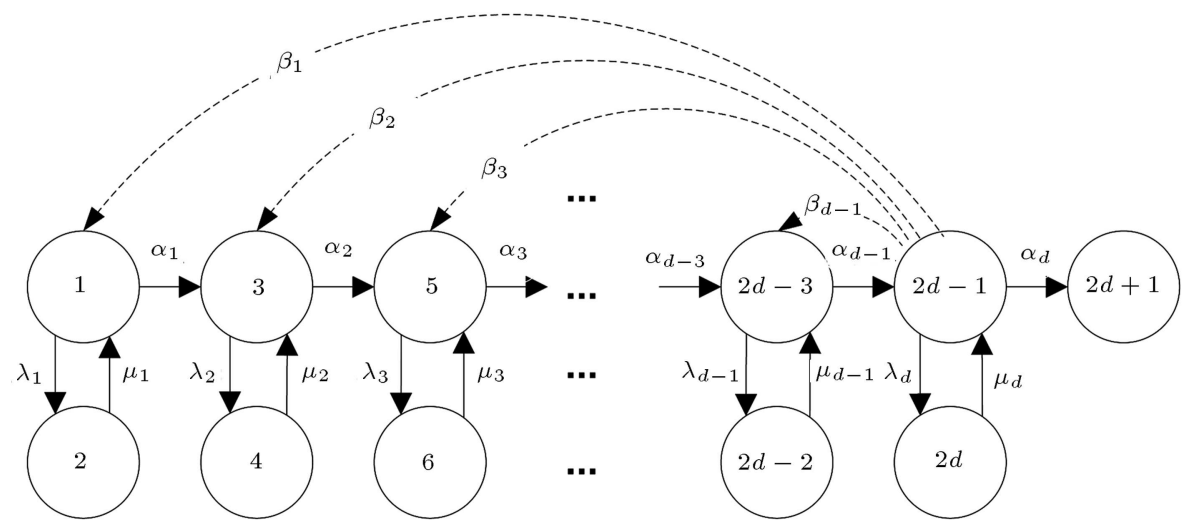

Figure 1. System state transition diagram. 
either deteriorate gradually and go to the first degraded state, or it may suddenly and randomly fail according to a Poisson process and go to a failed state. If the system fails, minimal repairs are carried out on it that restore the system to the previous state. When the system reaches the first degraded state, it may either go to the second degraded state upon degradation, or may go to a failed state from which a minimal repair is performed. The same process will continue for all acceptable degraded states. When the system reaches an unacceptable state, it cannot satisfy the customers' demand in a required performance level and must be treated as a failure. If the inspection finds the system in its last acceptable state (state $d$ ), a PM is carried out to restore the system to one of the higher performance states. The described process is considered as a Markov process; the system-state transition diagram is shown in Figure 1.

\subsection{Assumptions}

The considered assumptions in the proposed model are as follows:

1. System can have different levels of degradation corresponding to discrete performance rates, which vary from perfect state to completely failed state;

2. System can randomly fail from any operational state, and then a minimal repair is done on it;

3. The repair time is negligible compared to the system's useful life. Therefore, it does not enter into the calculations;

4. All transition rates are constant with the exponential distribution;

5. The current state of the system can be observed, and the time required for inspection is negligible. In other words, the inspection is carried out immediately.

\subsection{System description}

System-state transition can be described by the following notations and is illustrated in Figure 1:

$$
\text { (i): State }(i), i=1, \cdots, n \text { and } n=2 d+m
$$

State (1): Perfect functioning;

State $(2 j-1), j=2, \cdots, d$ : The system satisfies the customer demand with an acceptable performance level;

State $(2 d+1)$ : The system cannot satisfy the required demand after a degradation process and is in an unacceptable performance level;

State $(2 j), j=1, \cdots, d$ : The system encounters failure from an operational state and minimal repair must be carried out on it. $\lambda_{j}$ : $\quad$ Failure rate or the transition rate from state $(2 j-1)$ to the state $(2 j)$; $j=1, \cdots, d$;

$\mu_{j}$ : $\quad$ Minimal repair rate or transition rate from state $(2 j)$ to state $(2 j-1)$; $j=1, \cdots, d$;

$\alpha_{j}$ : Degradation rate or transition rate from state $(2 j-1)$ to state $(2 j+1)$; $j=1, \cdots, d$;

$\beta_{j}$ : The preventive maintenance transition rate from state $(2 d-1)$ to state $(2 j-1) ; j=1, \cdots, d-1$.

\section{The formulation of the problem}

As mentioned before, the proposed method aims to simultaneously optimize the total cost and the mean availability of the system throughout its useful lifetime. Next, the method of calculating the total cost function and the mean availability function are described.

\subsection{The total cost function}

The total cost is the sum of the costs of preventive maintenance and repair during the system life cycle. The variable $\psi_{j}$ is defined as a binary variable, so that it is 1 if preventive maintenance is carried out from state $(2 d-1)$ to state $(2 j-1, j=1, \cdots, d-1)$; otherwise, it is zero. $\mathrm{CPM}_{j}$ is the cost per unit time incurred if a PM is performed from state $(2 d-1)$ to $(2 j-1, j=1, \cdots, d-1) . p_{i}$ is the probability of being in the state $i$. It is assumed that the study period is given by the system life cycle $T$. Considering that $T$ is large enough, there exists a steady-state distribution of state probabilities. In this case, the expected PM cost of system is a function of the decision variable, $\psi_{j}$, and it is written as:

$$
E(\mathrm{CPM})=T \cdot p_{2 d-1} \sum_{j=1}^{d-1}\left(\mathrm{CPM}_{j} \times \psi_{j}\right) .
$$

Since in state $(2 d-1)$, a PM is certainly performed. Also, we have $\sum_{j=1}^{d-1} \psi_{j}=1$. In addition, $\mathrm{CR}_{j}$ is the cost per unit time incurred to repair system when it is in failed state $(2 j) ; j=1, \cdots, d$. Therefore, the expected repair cost can be defined as follows:

$$
E(\mathrm{CR})=T \sum_{j=1}^{d} \mathrm{CR}_{j} \cdot p_{2 j}
$$

Consequently, over the time interval $[0, T]$, the total cost of $\mathrm{PM}$ and repair is:

$$
T C=T\left[p_{2 d-1} \sum_{j=1}^{d-1}\left(\mathrm{CPM}_{j} \times \psi_{j}\right)+\sum_{j=1}^{d} \mathrm{CR}_{j} \cdot p_{2 j}\right] \text {. }
$$




\subsection{The availability function}

In Figure 1, each state $(2 j-1, j=1, \cdots, d-1)$ is characterized by a performance rate (or capacity) explained by $G_{2 j-1}$, ranging from the best performance rate $G_{1}$ to the lowest one $G_{2 d-1}\left(G_{1} \succ G_{2} \succ \cdots \succ\right.$ $\left.G_{2 d-1}\right)$. It should be noted that the performance rate of the failed states is zero (i.e., $G_{2 j}=0, j=1,2, \cdots, d$ ). Finally, the performance rate of the unacceptable state $(2 d+1)$ is less than customer demand. Since the performance rate at any time is a random variable, for the time interval $[0, T]$, the performance rate is a stochastic process. The probabilities associated with the various states at any time $t \geq 0$ are given by the set $P(t)=\left\{p_{1}(t), \cdots, p_{2 d+1}(t)\right\}$, where:

$$
p_{i}(t)=\operatorname{Pr}\left(G(t)=G_{i}\right), \quad i=1, \cdots, 2 d+1 .
$$

The system state acceptability depends on the relation between the system performance and the customer demand. This demand $W(t)$ is usually a random variable that changes over time and can take a discrete value of the set $W=\left\{W_{1}, \cdots, W_{2 d+1}\right\}$. It is assumed that demand is constant over time $(W(t)=W)$. As the system performance should exceed demand $W$, the acceptability function is defined as $F(G(t), W)=$ $G(t)-W[46]$. According to our notations, it is assumed that $G_{2 d+1}<W \leq G_{2 d-1}$. Instantaneous availability, $A(t)$, is the probability that the system performance level at the moment $t$ is greater than the customers' demand. In other word, system availability is the probability that the system is in one of acceptable states at time $t$.

$$
A(t)=\operatorname{Pr}(G(t) \geq W)=\sum_{j=1}^{d} p_{2 j-1}(t) .
$$

Since every system enters the absorbing state as $t$ becomes extremely large, the final state probabilities are as follows:

$$
\begin{aligned}
& \lim _{t \rightarrow \infty} p_{2 d+1}=1, \\
& \lim _{t \rightarrow \infty} p_{j}=0 \quad j=1, \cdots, 2 d .
\end{aligned}
$$

According to the proposed Markov model in Figure 1, Chapman-Kolmogorov equations [27] are used to calculate $p_{i}(t)$. These equations are as follows:

$$
\frac{d P_{1}(t)}{d t}=-\left(\alpha_{1}+\lambda_{1}\right) P_{1}(t)+\mu_{1} P_{2}(t)+\beta_{1} P_{2 d-1}(t) \psi_{1},
$$

$$
\begin{aligned}
\frac{d P_{2 j-1}(t)}{d t}= & -\left(\alpha_{j}+\lambda_{j}\right) P_{2 j-1}(t)+\mu_{j} P_{2 j}(t) \\
& +\alpha_{j-1} P_{2 j-3}(t)+\beta_{j} P_{2 d-1}(t) \psi_{j}
\end{aligned}
$$

for $j=2, \cdots, d-1$,

$$
\begin{aligned}
\frac{d P_{2 d-1}(t)}{d t}= & -\left(\beta_{j} \psi_{j}+\alpha_{d}+\lambda_{d}\right) P_{2 d-1}(t) \\
& +\mu_{d} P_{2 d}(t)+\alpha_{d-1} P_{2 d-3}(t)
\end{aligned}
$$

for $j$ corresponding to $\psi_{j}=1$,

$$
\begin{aligned}
& \frac{d P_{2 j}(t)}{d t}=-\mu_{j} P_{2 j}(t)+\lambda_{j} P_{2 j-1}(t) \\
& j=1,2, \cdots, d, \\
& \frac{d P_{2 d+1}(t)}{d t}=\alpha_{d} P_{2 d-1}(t),
\end{aligned}
$$

with the following initial values:

$$
p_{1}(t)=1, p_{2}(t)=p_{3}(t)=\cdots=p_{2 d+1}(t)=0 .
$$

Also, for each $t, 0 \leq t \leq T$, we have:

$$
\sum_{i=1}^{2 d+1} p_{i}(t)=1
$$

Thus, Eqs. (8)-(10) are the constraints of optimization problem for a particular time of $t$. The objective function is maximizing the mean availability over the useful life of the system. The mean availability function of the system during the useful lifetime of the system is written as follows:

$$
\bar{A}=\frac{\int_{t=0}^{T} A(t)}{T} .
$$

\subsection{The integration method}

In order to do simultaneous optimization of the system cost and availability, an integrated optimization approach is suggested based on the desirability function. The desirability function approach systematically transforms the objective function into a scale-free value called desirability. It assigns values between 0 and 1 to the possible values of objective function, in which desirability function gets 1 as the objective function gets its target value, and it will be zero when objective function lies outside its corresponding acceptable levels. Desirability function for system availability is shown in Eq. (12):

$$
d(\bar{A})= \begin{cases}\frac{\bar{A}-L_{A}}{U_{A}-L_{A}} & L_{A} \leq \bar{A} \leq U_{A} \\ 0, & \bar{A} \prec L_{A}\end{cases}
$$

where $\bar{A}$ is the obtained value for the system availability from Eq. (11), and $U_{A}$ can be set at the extreme value of the system availability:

$$
\begin{aligned}
& U_{A}=\max \bar{A}, \\
& \text { S.t. (Eqs. (8) to (10)). }
\end{aligned}
$$

$U_{A}$, determined by Eq. (13), represents the maximum possible value of system availability within a feasible 
region. $L_{A}$ is the lowest acceptable limit of system availability which can be determined on the basis of the decision-maker's subjective judgment. In other words, the degree of satisfaction of a decision-maker with respect to system availability is maximized when $\bar{A}$ equals its target value $\left(U_{A}\right)$ and decreases as $\bar{A}$ moves away from $U_{A}$. Finally, it will be zero when system availability is less than $L_{A}$.

Similarly, the desirability function of system cost is obtained from Eq. (14):

$$
d(T C)= \begin{cases}\frac{T C-U_{T C}}{L_{T C}-U_{T C}} & L_{T C} \leq T C \leq U_{T C} \\ 0 & T C \succ U_{T C}\end{cases}
$$

where $T C$ is the value obtained for the total cost function from Eq. (3), $L_{T C}$ is acquired by minimizing the total cost function in a feasible region, and $U_{T C}$ is asked from the decision-maker.

If a minimum operator is employed for aggregating the desirability functions of system cost and availability, an optimization problem can be stated as:

$\operatorname{Max} \lambda$.

Subject to:

$$
\begin{aligned}
& d(\bar{A}) \geq \lambda \\
& d(T C) \geq \lambda, \\
& \frac{d P_{1}(t)}{d t}=-\left(\alpha_{1}+\lambda_{1}\right) P_{1}(t)+\mu_{1} P_{2}(t)+\beta_{1} P_{2 d-1}(t) \psi_{1}, \\
& \frac{d P_{2 j-1}(t)}{d t}=-\left(\alpha_{j}+\lambda_{j}\right) P_{2 j-1}(t)+\mu_{j} P_{2 j}(t) \\
& +\alpha_{j-1} P_{2 j-3}(t)+\beta_{j} P_{2 d-1}(t) \psi_{j} \\
& \text { for } j=2, \cdots, d-1 \text {, } \\
& \frac{d P_{2 d-1}(t)}{d t}=-\left(\beta_{j} \psi_{j}+\alpha_{d}+\lambda_{d}\right) P_{2 d-1}(t) \\
& +\mu_{d} P_{2 d}(t)+\alpha_{d-1} P_{2 d-3}(t)
\end{aligned}
$$

for $j$ corresponding to $\psi_{j}=1$

$$
\begin{aligned}
& \frac{d P_{2 j}(t)}{d t}=-\mu_{j} P_{2 j}(t)+\lambda_{j} P_{2 j-1}(t) \\
& j=1,2, \cdots, d, \\
& \frac{d P_{2 d+1}(t)}{d t}=\alpha_{d} P_{2 d-1}(t), \\
& A(t)=\operatorname{Pr}(G(t) \geq w)=\sum_{j=1}^{d} p_{2 j-1}(t),
\end{aligned}
$$

$$
\begin{aligned}
& \sum_{i=1}^{2 d+1} p_{i}(t)=1, \\
& p_{i}(t) \geq 0 .
\end{aligned}
$$

This formulation aims to maximize the minimum satisfaction level of the decision-maker with respect to the system cost and the availability. It has several advantages over the existing methods.

Firstly, the majority of solution approaches in the literature assumed that there is no cost limitation or considered the cost limitation only as a constraint, whereas the proposed method optimizes total cost and system availability simultaneously.

Secondly, in contrast to other existing multiobjective approaches in literature, the suggested maximin approach is robust to the potential dependency between the total cost and availability of the system. In other words, the correlation between the objective functions will not affect the optimization process. As a result, existing aggregation approaches used can lead to misleading results for this problem.

Thirdly, the suggested maximin approach creates a better balance between the objective functions in comparison with other existing aggregation methods. In other words, many aggregation methods may lead to a decision variable vector that the value of some of the objective functions may not be incredible in the decision-makers' view. In the proposed approach, first, the acceptable range for each objective function is determined separately, and then it maximizes the overall satisfaction level within the ranges, such that the share of each objective function is properly reflected in the optimization process.

\section{Numerical example}

Consider a production system that has seven performance levels measured by hourly production rate, $G_{i}$. These production rates are presented in Table 1 . The customer demand, $W$, to be satisfied is 500 parts per hour. The Markov model of this multi-state system is illustrated in Figure 2.

According to the Markov model, there are two possible preventive maintenance actions. It is assumed that the transition rate of perfect $\mathrm{PM}\left(\beta_{1}\right)$ is equal to 0.08 , and the transition rate of imperfect PM $\left(\beta_{2}\right)$ is 0.02 . The other used parameters are presented in Table 2.

The solution space of this problem has two cases which are:

Table 1. Hourly production rate for each state.

\begin{tabular}{cccccccc}
\hline State $\boldsymbol{i}$ & 1 & 2 & 3 & 4 & 5 & 6 & 7 \\
\hline $\boldsymbol{G}_{\boldsymbol{i}}$ & 1000 & 0 & 750 & 0 & 500 & 0 & 0 \\
\hline
\end{tabular}




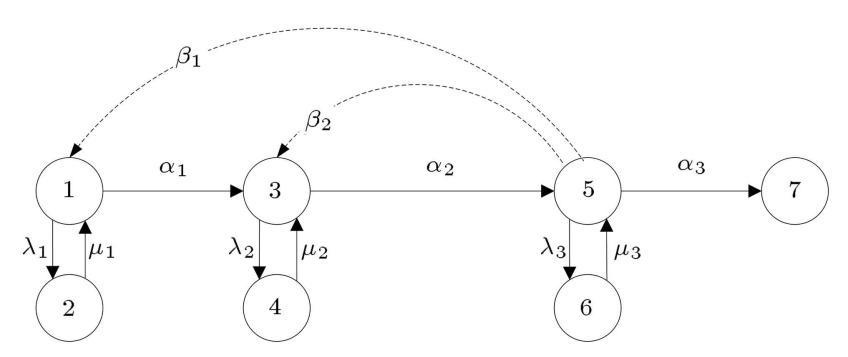

Figure 2. System state transition diagram in a numerical example.

Table 2. Transition rates for the numerical example.

\begin{tabular}{ccccccccc}
\hline $\boldsymbol{\alpha}_{\mathbf{1}}$ & $\boldsymbol{\alpha}_{\mathbf{2}}$ & $\boldsymbol{\alpha}_{\mathbf{3}}$ & $\boldsymbol{\lambda}_{\mathbf{1}}$ & $\boldsymbol{\lambda}_{\mathbf{2}}$ & $\boldsymbol{\lambda}_{\mathbf{3}}$ & $\boldsymbol{\mu}_{\mathbf{1}}$ & $\boldsymbol{\mu}_{\mathbf{2}}$ & $\boldsymbol{\mu}_{\mathbf{3}}$ \\
\hline 0.03 & 0.05 & 0.07 & 0.005 & 0.008 & 0.01 & 0.01 & 0.02 & 0.04 \\
\hline
\end{tabular}

Table 3. The cost per hour incurred to repair system.

\begin{tabular}{cccc}
\hline $\boldsymbol{j}$ & 1 & 2 & 3 \\
$\mathbf{C R}_{\boldsymbol{j}}$ & 5.3 & 3.8 & 4.5 \\
\hline
\end{tabular}

Table 4. Preventive maintenance cost incurred per hour.

\begin{tabular}{ccc}
\hline $\boldsymbol{j}$ & 1 & 2 \\
$\mathrm{CPM}_{\boldsymbol{j}}$ & 2.3 & 1.4 \\
\hline
\end{tabular}

- $\psi_{1}=1, \psi_{2}=0$,

- $\psi_{1}=0, \psi_{2}=1$.

In order to evaluate the system availability, first, Chapman-Kolmogorov equations are solved for the mentioned Markov model to obtain the probabilities $P_{i}(t),(i=1, \cdots, 7)$ separately in both cases. Then, the system availability at time $t$ is calculated using $A(t)=P_{1}(t)+P_{3}(t)+P_{5}(t)$. Finally, assuming that the useful life of the system is determined 80 hours, system availability $(\bar{A})$ and its corresponding desirability $(d(\bar{A}))$ can be obtained by Eqs. (11) and (12), respectively.

The repair costs and the preventive maintenance costs are presented in Tables 3 and 4, respectively.

The expected repair and PM costs and the total cost will be calculated using Eqs. (1), (2), and (3), respectively. Then, the desirability function of the total cost and system availability will be achieved by assuming that the lower bound of acceptability for the system availability $\left(L_{A}\right)$ and the upper bound of acceptability for the total cost $\left(U_{T C}\right)$ are 0.5 and 80 , respectively. After this step, the final model can be written as follows:

$\operatorname{Max} \lambda$

Subject to : $\quad d(A) \geq \lambda, \quad d(T C) \geq \lambda$, $\frac{d P_{1}(t)}{d t}=-\left(\alpha_{1}+\lambda_{1}\right) P_{1}(t)+\mu_{1} P_{2}(t)+\beta_{1} P_{5}(t) \psi_{1}$

$$
\begin{aligned}
\frac{d P_{3}(t)}{d t}= & -\left(\alpha_{2}+\lambda_{2}\right) P_{3}(t)+\mu_{2} P_{4}(t)+\alpha_{1} P_{1}(t) \\
& +\beta_{2} P_{5}(t) \psi_{2} \\
\frac{d P_{5}(t)}{d t}= & -\left(\beta_{j} \psi_{j}+\alpha_{3}+\lambda_{3}\right) P_{5}(t)+\mu_{3} P_{6}(t) \\
& +\alpha_{2} P_{5}(t)
\end{aligned}
$$

for $j$ corresponding to $\psi_{j}=1$,

$$
\begin{aligned}
& \frac{d P_{2}(t)}{d t}=-\mu_{1} P_{2}(t)+\lambda_{1} P_{1}(t), \\
& \frac{d P_{4}(t)}{d t}=-\mu_{2} P_{4}(t)+\lambda_{2} P_{3}(t), \\
& \frac{d P_{6}(t)}{d t}=-\mu_{3} P_{6}(t)+\lambda_{3} P_{5}(t), \\
& \frac{d P_{7}(t)}{d t}=\alpha_{3} P_{5}(t), \\
& A(t)=P_{1}(t)+P_{3}(t)+P_{5}(t), \\
& \sum_{i=1}^{7} p_{i}(t)=1, \\
& p_{i}(t) \geq 0 .
\end{aligned}
$$

After solving the above mathematical programming using MATLAB software package, the optimum solution is achieved as $\psi_{1}=1, \psi_{2}=0$.

Subsequently, this $\psi_{1}=0, \psi_{2}=0$ means that during its useful life, whenever this system reaches the state 3 , the perfect preventive maintenance is carried out; the system is restored to the perfect functioning state in this way.

\section{Conclusion}

This paper considered a multi-state degraded system in which status of system is considered to degrade over time, and this degradation may affect system performance. In other words, as time progresses, system may either deteriorate gradually and go to lower performance state, or it may suddenly and randomly fail according to a Poisson process called Poisson failure. If the system fails, minimal repairs are carried out to restore the system to its previous state. When the inspections showed that the system has reached its last acceptable state, a PM is carried out to restore the system to one of the higher performance states, varying from minor maintenance to major maintenance. A minor PM restores the system to the previous degraded state, while a major PM restores it to the "as good as new" state. To find the optimal PM level, such that the mean availability of the system is maximized and 
the total cost of the system is minimized, an integrated optimization approach is suggested based on the desirability function approach. The suggested aggregation method is robust to the potential dependency between the total cost and the mean availability. It also ensures that both objective functions fall in decision-maker's acceptable region.

As a direction for future research, the proposed model can be extended for a joint redundancy and imperfect preventive maintenance planning optimization problem for series-parallel multi-state degraded systems.

\section{References}

1. Jiang, Y., Chen, M. and Zhou, D. "Joint optimization of preventive maintenance and inventory policies for multi-unit systems subject to deteriorating spare part inventory", Journal of Manufacturing Systems, 35(1), pp. 191-205 (2015).

2. Yuan, W.Z. and Xu, G.Q. "Modelling of a deteriorating system with repair satisfying general distribution", Applied Mathematics and Computation, 218(11), pp. 6340-6350 (2012).

3. Kumar, A., Saini, M., and Malik, S.C. "Performance analysis of a computer system with imperfect fault detection ofhHardware", Procedia Computer Science, 45(0), pp. 602-610 (2015).

4. Samad, M. "An efficient algorithm for simultaneously deducing MPs as well as cuts of a communication network", Microelectronics Reliability, 27(1), pp. 437441 (1987).

5. Doulliez, P. and Jalnoulle, E. "Transportation network with random arc capacities", RAIRO, 3(1), pp. 45-60 (1972).

6. Aven, T. "Availability evaluation of oil/gas production and transportation systems", Reliability Engineering, 18(1), pp. 35-44 (1987).

7. Billinton, R. and Allan, R., Reliability of Power Systems, London, Pitman (1984).

8. Faghih-Roohi, S., Xie, M., Ming Ng, K. and Yam, R.C.M. "Dynamic availability assessment and optimal component design of multi-state weighted k-out-ofn systems", Reliability Engineering \& System Safety, 123(0), pp. 57-62 (2014).

9. Sheu, S.-H., Chang, C.C., Chen, Y.L. and Zhang, Z.G. "Optimal preventive maintenance and repair policies for multi-state systems", Reliability Engineering \& System Safety, 140(0), pp. 78-87 (2015)

10. Li, Y.F. and Peng, R. "Availability modeling and optimization of dynamic multi-state series-parallel systems with random reconfiguration", Reliability Engineering \& System Safety, 127(0), pp. 47-57 (2014).

11. Murchland, J. "Fundamental concepts and relations for reliability analysis of multistate systems, reliability and fault tree analysis", Theoretical and Applied Aspects of System Reliability, Philadelphia: SIAM, 3(1), pp. 581-618 (1975).

12. Barlow, S. and Wu, A. "Coherent systems with multistate components", Mathematics of Operations Research, 3(1), pp. 275-281 (1978).

13. El-Neweihi, E., Proschan, F. and Sethuraman, J. "Multistate coherent systems", Journal of Applied Probability, 15(12), pp. 675-688 (1978).

14. Ross, S.M. "Multi valued state component systems", Annals of Probability, 7(2), pp. 379-83 (1979).

15. Lisnianski, A. and Levitin, G., Multi-State System Reliability, Assessment, Optimization and Applications, Singapore, World Scientific Publishing Co. Pte. Ltd (2003).

16. Yingkui, G. and Jing, L. "Multi-state system reliability: A new and systematic review", Procedia Engineering, 29(1), pp. 531-536 (2012).

17. Zio, E. and Podofillini, L. "A Monte Carlo approach to the estimation of importance measures of multi-state components", Reliability and Maintainability Annual Symposium (RAMS), pp. 129-34 (2004).

18. Xue, J. and Yang, K. "Dynamic reliability analysis of coherent multi-state systems", IEEE Transactions on Reliability, 44(1), pp. 683-688 (1995).

19. Pham, H., Suprasad, A. and Misra, R. "Availability and mean life time prediction of multistage degraded system with partial repairs", Reliability Engineering \& System Safety, 56(1), pp. 169-73 (1997).

20. Ushakov, I. "Universal generating function", Soviet Journal of Computing and System Science, 24(5), pp. 118-29 (1986).

21. Levitin, G., Universal Generating Functionin Reliability Analysis and Optimization, Berlin (Heidelberg/New York): Springer-Verlag (2003).

22. Peng, R., Mo, H., Xie, M. and Levitin, G. "Optimal structure of multi-state systems with multi-fault coverage", Reliability Engineering \& System Safety, 119(2), pp. 18-25 (2013).

23. SU, C.-T. and Chang, C. "Minimization of the life cycle cost for a multi state system under periodic maintenance", International Journal of Systems Science, 31(2), pp. 217-27 (2000).

24. Zhou, Y., Zhang, Z., Lin, T.R. and Ma, L. "Maintenance optimisation of a multi-state series-parallel system considering economic dependence and statedependent inspection intervals", Reliability Engineering \& System Safety, 111(1), pp. 248-259 (2013).

25. Zhang, Y., Yam, R. and Zuo, J. "Optimal replacement policy for a multistate repairable system", Journal of the Operational Research Society, 53(1), pp. 336-41 (2002). 
26. Lam, Y. "A monotone process maintenance model for multistate system", Journal of Applied Probability, 42(1), pp. 1-14 (2005).

27. Soro, I., Nourelfath, M. and Ait-Kadi, D. "Performance evaluation of multi-state degraded systems with minimal repairs and imperfect preventive maintenance", Reliability Engineering \& System Safety, 95(2), pp. 65-69 (2010).

28. Nourelfath, M., Chatelet, E. and Nahas, N. "Joint redundancy and imperfect preventive maintenance optimization for series-parallel multi-state degraded systems", Reliability Engineering and System Safety, 103(1), pp. 51-60 (2012).

29. Aggarwal, K. "A simple method for reliability evaluation of a communication system", IEEE Transactions on Communications, 23(1), pp. 563-565 (1975).

30. Ke, W. and Wang, S. "Reliability evaluation for distributed computing networks with imperfect nodes", IEEE Transactions on Reliability, 46(1), pp. 342-349 (1997).

31. Aven, T. "Some considerations on reliability theory and its applications", Reliabiality Engingineering \& System Safety, 21(3), pp. 215-223 (1988).

32. Colbourn, C., The Combinatorics of Network Reliability, New York, Oxford University Press (1987).

33. Hoyland, A. and Rausand, M., System Reliability Theory: Models and Statistical Methods, New York, Wiley (1994).

34. Yeh, W. "A revised layered-network algorithm to search for all d-minpaths of a limited-flow acyclic network", IEEE Transactions on Reliability, 47(3), pp. 436-442 (1998).

35. Yeh, W. "Search for MC in modified networks", Computers \& Operations Reseasrch, 28(2), pp. 177184 (2001).

36. Yeh, W. "A simple algorithm to search for alldMPs with unreliable nodes", Reliab. Engin. Sys. Safe, 73(1), pp. 49-54 (2001).

37. Yeh, W. "Search for all d-mincuts of a limited-flow network", Computers \& Operations Reseasrch, 29(13), pp. $1843-1858$ (2002).

38. Huang, H. "Fuzzy multi-objective optimization decisionmaking of reliability of series system", Microelectronics Reliability, 37(3), pp. 447-449 (1997).

39. Lin, J. "Evaluating the reliability of limited-flow networks under the cost constraint", IIE Trans, 30(3), pp. 1175-1180 (1998).

40. Majety, S., Dawande, M. and Rajgopal, J. "Optimal reliability allocation with discrete cost-reliability data for components", Operations Research, 47(6), pp. 899906 (1999).
41. Jahromi, E., Salmani. M.H. and Ghasemi, F. "A fourphase algorithm to improve reliability in series-parallel systems with redundancy allocation", Scientia Iranica, 21(3), pp. 1072-1082 (2014).

42. Amiri, M. and Ghasemi F. "A methodology for analyzing the transient reliability of systems with identical comp onents and identical repairmen", Scientia Iranica, 14(2), pp. 72-77 (2007).

43. Fazlollahtabar, F. and Jalali, S.G. "Adapted Markovian model to control reliability assessment in multiple AGV", Scientia Iranica, 20(6), pp. 224-227 (2013).

44. Kianfar, F. "Optimal planning of equipment maintenance and replacement on a variable horizon", Scientia Iranica, 11(3), pp. 185-192 (2004).

45. Aven, T. "On performance measures for multistate monotone systems", Reliability Engineering \& System Safety, 41(2), 259-266 (1993).

46. Mokhtari, H., Mozdgir, A. and Nakhai Kamal Abadi, I. "A reliability/availability approach to joint production and maintenance scheduling with multiple preventive maintenance services", International Journal of Production Research, 50(20), pp. 5906-5925 (2012).

\section{Biographies}

Ali Salmasnia is currently an Assistant Professor in University of Qom, Qom, Iran. His research interests include quality engineering, multi-response optimization, applied multivariate statistics, and multi-criterion decision making. $\mathrm{He}$ is the author or co-author of various papers published in Applied Soft Computing, Neurocomputing, IEEE Transactions on Engineering Management, International Journal of Advanced Manufacturing Technology, Applied Mathematical Modelling, Expert Systems with Applications, International Journal of Information Technology and Decision Making, and TOP.

Ehsan Ameri received his BS degree from the Islamic Azad University and his MS degree in Industrial Engineering from the Tarbiat Modares University in Iran. His current research interests include reliability, healthcare, and multi-criteria decision making.

Ali Ghorbanian is a Lecturer in the department of Industrial Engineering in the Esfarayen University of Technology, North Khorasan, Iran. He holds master's degree of Industrial Engineering from the Tarbiat Modares University (TMU). His research interests are decision support systems, simulation, RSM, knowledge management, and process management.

Hadi Mokhtari is currently an Assistant Professor of Industrial Engineering in University of Kashan, Iran. His current research interests include the applications of operations research and artificial intelligence techniques to the areas of project scheduling, production 
scheduling, and supply chain management. He also published several papers in international journals such as Computers and Operations Research, International Journal of Production Research, Applied Soft Com- puting, Neurocomputing, International Journal of Advanced Manufacturing Technology, IEEE Transactions on Engineering Management, and Expert Systems with Applications. 